\title{
A method of dual-threshold dynamic image segmentation based on DTBNN algorithm *
}

\author{
Song Lihua, Fu Yongqiang, Li Yebai \\ Department of Computer Sciences \\ North China University of Technology \\ Beijing 100144, China \\ fred_fu612@163.com
}

\begin{abstract}
In order to deal with the segment problem of dynamic image which has indistinctive ups and downs feature in histogram, a method of dual-threshold dynamic image segmentation based on DTBNN (Decision Tree Based on Neutral Network) algorithm is proposed. Firstly, according to the correspondence between decision tree and neural network to build a stable and efficient training neural network; Then, work out the mean of gray value, the maximum deviation and the threshold mapping function as sample data to train the neural network; Finally, using the trained neural network to get threshold mapping function by testing images, and achieve dualthreshold image segment using the result of the upper and lower threshold values which are calculate from previous step. The simulation results shows that this method does not depend on the feature of histogram and it could accurately get upper and lower segmentation threshold. Compared with OTSU dual-threshold method and maximum entropy dual-threshold method, the proposed method could achieve better dynamic image dualthresholds segmentation.
\end{abstract}

Keywords-dual-thresholds; image segmentation; neural network; decision tree

\section{INTRODUCTION}

The main technique point of image segmentation is how to separate out recognized objects from image background. In generally, there are several methods to do image segmentation such as based on threshold segmentation method, based on edge extracting segmentation method and based on region segmentation method. Based on threshold image segmentation method is the most commonly method among all of these methods which have mentioned above, because this algorithm is simple, efficient and widely used in image processing. Threshold segmentation method could be divided into two segmentation method, global threshold segmentation and local threshold segmentation; the local threshold segmentation method involving algorithm contains maximum between-class variance method (also known as OTSU method), maximum entropy method and fuzzy entropy method. In these image segmentation algorithms, we could use single threshold or multi-threshold to process image segmentation. Since single threshold image segmentation method usually use one threshold to divide proto-image into two parts with grey of image, so could not use this method to efficiently segmented target objects from the image normally. Dual-threshold image

Chinese National Natural Science Foundation (61300171, 61371143); Beijing Higher Education Young Elite Teacher Project (YETP1419); the Project of Construction of Innovative Teams and Teacher Career Develop ment for Universities and Colleges under Beijing Municipality (IDHT20130502). segmentation method used by dividing the upper and the lower limits to extract two thresholds middle gray field as an availability region, it will be able to more efficiently segment target objects from the image.

In dual-threshold image segmentation algorithm, one of based on OTSU algorithm of dual-threshold image segmentation and improved algorithm[1][2] more commonly be used, but OTSU algorithm has certain requirements for feature of histogram. In image which has three peeks feature of histogram, this algorithm has better segmentation results, while poor segmentation result in other situation. Base on maximum entropy algorithm of dual-threshold image segmentation and improved algorithm[3], because of it according to the total maximum entropy between target object and background, so the image processing need to have three peeks value in the histogram feature as well. Base on fuzzy entropy algorithm of dual-threshold image segmentation and improved algorithm[4][5], using upper and lower threshold to separate image into three parts: dark, gray, bright, each part of scope is determined by the membership functions. Because it is through membership function to determine the bandwidth of two fuzzy areas, therefore it could not effectively determine membership function in dynamic image. So it could not be used for dynamic image segmentation and has poor flexibility in applications. In addition, based on dichotomy algorithm of multi-threshold image segmentation [6], this method is still based on OTSU algorithm, and divide target threshold depends on histogram peek feature as well.

In the actual project mentioned in this paper, it is necessary to extract effective feature information from image of rotating objects. Due to image is formatted by light reflection and environment light through rotating object surface will format different imaging with different rotation, leading to the collection image have large variant in histogram and there is no obvious peek feature in histogram sometimes. According to the necessary of actual project and application fields above mentioned, this paper propose a method of dual-threshold dynamic image segmentation based on DTBNN algorithm, the method show a well result of image segmentation in practical applications. 


\section{DTBNN ALGORITHM}

Artificial neural network has been widely used in identification and classification algorithms. Because it could be through divisions learning or no division learning way to achieve an effect classifier which is similar to biological neutral network to processing information and classification. In this paper, we employed back propagation neural network, that base on forward neural network and add reverse learning ability on the forward neural network. This back propagation neural network model is the most widely applied currently also, we use neural network to call it for short in this paper. This model is multi-layer forward feedback network according to error reverse propagation; the neural network topology includes input layer, hidden layer and output layer. By the steepest descent method learning makes neural network store a large of the mapping relationships from input layer to output layer; and using these mapping relationships which in established neural network could make reasoning and classification for input information. Classification technology of image recognition is also often use neural network, such as Face Recognition [7], Classification and Selection [8] and so on. All of them use already segmented image feature to classify and to meet the recognition requirements, but in this paper propose that after learned and trained neural network apply to classification and selection of image threshold function in the processing.

Because in the actual application, neural network's structure and designing of its initial parameter are more difficult and serious impact on neural network performance. After depth study of entropy network which Sethi proposed [9], Li proposed a neural network algorithm base on Decision Tree (DTBNN) [10]; and through the "exclusive or" problem and multi-classification issue to verify the feasibility of this algorithm and analysis its effectiveness. Decision tree algorithm and neural network algorithm are two different mechanisms of learning classification, the former learning classification use attributes of decision objects, the later learning classification base on weighted relationship among neurons. Although the two mechanisms are not same completely, but there is certain correspondence between decision tree and neural network. In DTBNN algorithm what Li proposed, make correspond between internal node number of decision tree and input layer neurons number of neural network; the number of leaf nodes in decision tree corresponding to the number of hidden layer neurons in neural network; the number of categories in decision tree corresponding to the number of output layer neurons in neural network; make correspond between connection of decision tree and neuronal connection of neural network; the connection weight of decision tree corresponding to connection weight of neural network. Fig. 1 show the correspondence relationship between decision tree and neural network, the rectangle of the left side represent part of decision tree and the ellipse of the right side represent part of neural network.

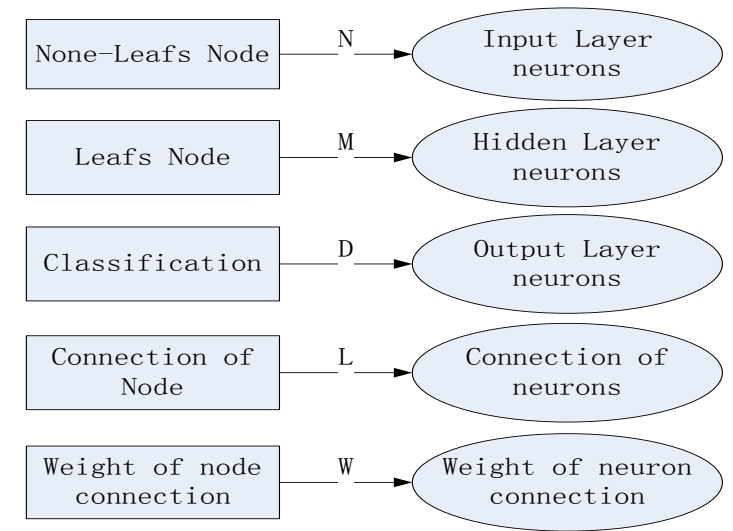

Fig. 1. The correspondence rule between decision tree and neural network

According to the correspondence rules between decision tree and neural network that was shown in Figure 1,we could use the decision tree to construct hierarchical structure of neural network and to initialize the parameter of neural network. $\mathrm{N}$ internal nodes of decision tree correspond to $\mathrm{N}$ input layer neurons of neural network; $\mathrm{M}$ leaf node of decision tree correspond to $\mathrm{M}$ hidden layer neurons of neural network; $\mathrm{D}$ kinds of classification strategy of decision tree correspond to D output layer neurons of neural network; L internal node connections of decision tree correspond to $\mathrm{L}$ neurons connections of neural network; w the connection weight of decision tree correspond to $\mathrm{w}$ the neuron connection weight of neural network.

Since the neuron number of hidden layer in DTBNN algorithm is determined by the leaf node of decision tree, it lead to overly complexity and make neural network easy going to over-fitting when build up hidden layer neurons relationship. Therefore, Liu through deeply study the principle of information entropy of maximum gain, then proposed the number of hidden layer neuron could be determined by depth of decision tree in DTBNN algorithm [11]. it means that there is the lengthier conjunctive chain from root node to leaf node in a decision tree, and the length of this chain is the depth of the decision tree. When the number of hidden layer neuron in neural network depends on the length of maximum conjunctive chain in corresponding decision tree, the neural network has best performance. And this neural network neither appear incorrect classification by less neurons, nor appear over-fitting problems due to excessive neuron. Liu through "exclusive or problems" and "classification of medical issues" these two experiments verified the validity of this improved algorithm.

According to the neural network construction method base on decision tree which proposed by $\mathrm{Li}$ and Liu, after appropriately improved construction method of DTBNN in this paper, applied this construction method in neural network of image threshold segmentation processing. Therefore, the correspondence rules between decision tree and neural network proposed in this paper: (1) the number of branches for decision tree correspond to the number of input layer neurons of neural network; (2) the depth of decision tree correspond to the number of hidden layer neurons of neural network; (3) the number of classification in decision tree correspond to the 
number of output layer neurons of neural network; (4) connections of decision tree correspond to connections of neural network.

\section{DuAL-THRESHOLD IMAGE SEGMENTATION BASED ON DTBNN}

\section{A. Dual-threshold image segmentation}

Let the size of processed image is $X \times Y$, the gray value $f(x$, $y$ ) takes $0,1,2 \ldots L-1, L$ means the gray level of image, taking 256 levels in this paper. Supposed

$$
\begin{aligned}
& \mathrm{D}_{\mathrm{i}}=\{(\mathrm{x}, \mathrm{y}): \mathrm{f}(\mathrm{x}, \mathrm{y})=\mathrm{i},(\mathrm{x}, \mathrm{y}) \in \mathrm{D}\}, \\
& \mathrm{h}_{\mathrm{i}}=\mathrm{n}_{\mathrm{i}} / \mathrm{N}
\end{aligned}
$$

where $\mathrm{D}$ is the whole set domain of measured image pixel, $i=0,1, \ldots, L-1$ are gray levels, $D_{i}$ represent the set of pixel whose gray level belong to $i, n_{i}$ represent the number of image pixel in $\mathrm{D}_{\mathrm{i}}$ domain, $N=X \times Y$ is the gross number of pixel, $\mathrm{h}_{\mathrm{i}}$ is the probability distribution of gray value is $i$.

$$
\mathrm{H}=\left\{\mathrm{h}_{0}, \mathrm{~h}_{1}, \ldots, \mathrm{h}_{\mathrm{L}-1}\right\}
$$

represent histogram of processed image.

This paper put dual-threshold to use segmentation image, let two thresholds are $t_{1}$ and $t_{2}$, then divide image domain $D$ into $D_{l}, D_{t}, D_{m} . D_{l}$ is the non-target domain, is the part of low gray level; $D_{t}$ is the target domain, is the part of gray level compiling with the threshold rang; $D_{m}$ is the non-target domain, is the part of high gray level. i.e.

$$
\begin{aligned}
& \boldsymbol{D}_{\boldsymbol{l}}=\left\{(\mathrm{x}, \mathrm{y}): \mathrm{f}(\mathrm{x}, \mathrm{y}) \leq \mathrm{t}_{1},(\mathrm{x}, \mathrm{y}) \in \mathrm{D}\right\}, \\
& \boldsymbol{D}_{\boldsymbol{t}}=\left\{(\mathrm{x}, \mathrm{y}): \mathrm{t}_{1}<\mathrm{f}(\mathrm{x}, \mathrm{y}) \leq \mathrm{t}_{2},(\mathrm{x}, \mathrm{y}) \in \mathrm{D}\right\}, \\
& \boldsymbol{D}_{\boldsymbol{m}}=\left\{(\mathrm{x}, \mathrm{y}): \mathrm{t}_{2}<\mathrm{f}(\mathrm{x}, \mathrm{y}),(\mathrm{x}, \mathrm{y}) \in \mathrm{D}\right\} .
\end{aligned}
$$

Then the probability distribution of gray level is (1)

$$
\begin{aligned}
& H_{l}=H\left(\boldsymbol{D}_{l}\right)=\sum_{i=0}^{\mathrm{t}_{1}} h_{\mathrm{i}}, \\
& H_{t}=H\left(\boldsymbol{D}_{\boldsymbol{t}}\right)=\sum_{i=\mathrm{t}_{1}}^{\mathrm{t}_{2}} h_{\mathrm{i}}, \\
& H_{m}=H\left(\boldsymbol{D}_{\boldsymbol{m}}\right)=\sum_{i=\mathrm{t}_{2}}^{\mathrm{L}-1} h_{\mathrm{i}} .
\end{aligned}
$$

Moreover, we could obtain two basic characteristics from the image histogram: Means of image gray and maximum deviation of image gray, representing with $M$ and $S$. Means of image gray $M$ is the sum of products between total gray level and probability distribution, performance the trend of the overall image gray in histogram, and the value is smaller the overall bright of image move to dark side, otherwise it move to light. Maximum deviation of image $S$ is the maximum difference between any gray of image and the means of image gray, performance the difference between the peek value or the valley value and mean of gray in the histogram, which reflect the change degree of light and dark. Calculate as (2):

$$
\begin{aligned}
& M=\sum_{i=0}^{\mathrm{L}-1}\left(i * h_{\mathrm{i}}\right)=\frac{1}{\mathrm{~N}} \sum_{i=0}^{\mathrm{L}-1}\left(i * \mathrm{n}_{\mathrm{i}}\right), \\
& S=\operatorname{MaX}_{0 \leq \mathrm{i} \leq \mathrm{L}-1}\{(i-M)\} \wedge\left(h_{i} \neq 0\right) .
\end{aligned}
$$

Because the $M$ and the $S$ individually represent the trend of image gray changes and relative diversity difference in image, for the segmentation image in project, according to the changes of its $M$ and $S$ could adopt different dual-threshold to segment. In other words, there is a function mapping $T=G(m$, $\mathrm{s})$, where $\mathrm{T}$ is one-dimensional vector composited by $\mathrm{t}_{1}$ and $\mathrm{t}_{2}$, besides meet the condition $t_{1}<t_{2}, G(m, s)$ is the mapping set of relation function, $\mathrm{m}$ is the gray mean value of measured image and satisfied $\mathrm{m} \in[0,255], \mathrm{s}$ is the maximum gray difference in measured image and satisfied $s \in[0,255)$. the mapping relation set $\mathrm{G}$ as (3):

$$
\left\{\begin{array}{rcrl}
G_{1}(m, s) & =T_{1} ; & (0 \leq m<10,0 \leq s<10) \\
G_{2}(m, s)=T_{2} ; & (0 \leq m<10,10 \leq s<20) \\
\ldots & \ldots \\
G_{26}(m, s)=T_{26} ; & (0 \leq m<10,250 \leq s<255) \\
\ldots & \ldots \\
G_{676}(m, s)=T_{676} ; & (250 \leq m<255,250 \leq s<255)
\end{array}\right.
$$

In them, the $\mathrm{m}$ and the $\mathrm{s}$ with 10 increments gradient are divided into 26 rang of fields, then the number of combined categories by $\mathrm{m}$ and $\mathrm{s}$ is $676, \underset{1 \leq i \leq 676}{G_{i}}(m, s)$ represent the relation mapping function under categories of $i, T_{i}$ represent the threshold vector under categories of $i$. Due to the limited space of this paper and that it is not point which wants to present in this paper, the explicit mapping function not list at here.

\section{B. Construct DTBNN}

Based on the DTBNN algorithm which Li and Liu studied, this paper proposed a DTBNN construction method for dualthreshold image segmentation. DTBNN construction steps designed by this paper as follow.

1) According to the corresponding rules between decision tree and neural network, determine the number of the layers of neural network, input layer and output layer neurons.

2) Determining the number of hidden layer neurons of neural network by maximum conjunctive chain.

3) Following the rules set initial value of network parameters.

4) Add connection to each neuron of neural network.

5) Using samples train neural network.

According to (3) see that, the corresponding categories relationship formed by the mean of image gray $M$, the maximum gray difference $S$ and the image segmentation mapping function $G$. By this categories relationship could build up a multi-branch classification decision tree, logic 
indicated in Fig. 2, $M$ is mean of image gray, $S$ is the maximum deviation of image gray, $m_{i}$ is the $i$-Class range domain of gray mean, $s_{i}$ is the $\mathrm{i}$-Class range domain of maximum gray deviation, $G_{i}$ is the i-Class mapping function. Round represent the internal determine node of decision tree, $M$ and $S$ are the condition of determining branch in each range domain; Square represent the classification information of decision tree. According to the image characteristic in project, retain the branch of satisfied condition $\left\{G_{i}(m, s): 50 \leq m<200\right.$, $20 \leq \mathrm{s}<150\}$, and cut off other branch for reducing the redundancy of the classification decision tree.

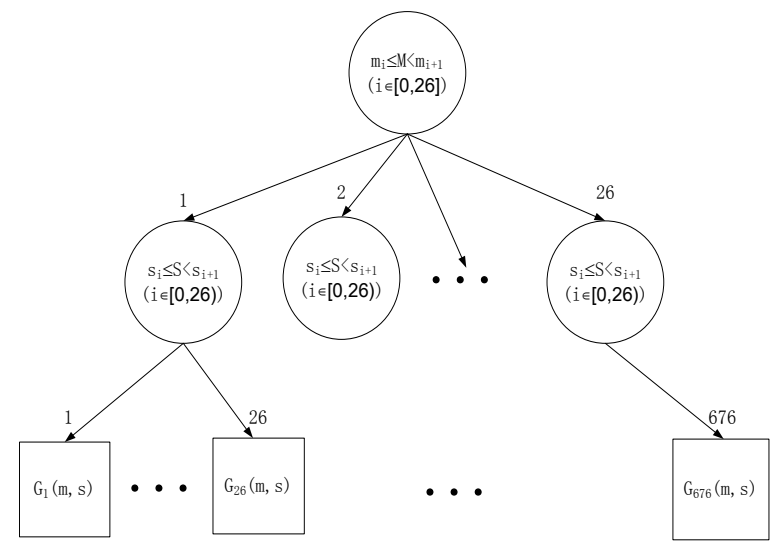

Fig. 2. The logic structure of classification decision tree

By the classification decision tree shown in Fig. 2, after pruning algorithm and combining mapping relation, finally build up the less redundancy classification decision tree. Accordance with the corresponding rule between decision tree and neural network, we could design for three layer neural network, which are input layer, hidden layer and output layer. The number of neurons in input layer is equal to the determine condition in the decision tree, in this paper is 26; the number of neurons in output layer is equal to the classification number of decision tree, in this paper after pruning and combining it is 18; the number of neurons in hidden layer is the length of maximum conjunctive chain which is the depth of the decision tree also, in this paper is 10. Through the decision tree constructing the neural network shown its logic indicate as Fig. 3 , where $W_{i}$ is the connection weight from input layer neuron to hidden layer neuron, $W_{0}$ is the connection weight from hidden layer neuron to output layer neuron, $m$ is the mean value of measured image gray and is the input parameter, $s$ is the maximum gray deviation of measured image and is the input parameter also, $t_{1}$ and $t_{2}$ are the necessary upper and lower threshold of image segmentation.

In this neural network, the connection weight $\mathrm{W}_{\mathrm{i}}$ from input layer to hidden layer is $26 \times 10$ matrix, its initialization rules are: supposed that the basic weight is $\omega_{i}$, in matrix $W_{i}$ the connection weight which were cut off are $-\omega_{\mathrm{i}}$ and others are $\omega_{\mathrm{i}}$. The connection weight $\mathrm{W}_{\mathrm{o}}$ from hidden layer to output layer is $10 \times 18$ matrix, its initialization rules are: supposed that the basic weight is $\omega_{0}$, in matrix $\mathrm{W}_{\mathrm{o}}$ the connection weight are $\omega_{0}$ of all. According to the proof of initial setting rule by Li[10] , this method has better learning performance and convergence than random setting two connection matrix initial value, in this paper set basic weight $\omega_{\mathrm{i}}$ and $\omega_{\mathrm{o}}$ to $\omega_{\mathrm{i}}=\omega_{0}=4$. Add the connections which take connection weight into neurons of each layer, it connects the separate hierarchical structure to the weighted connected network, become the initial neural network at last.

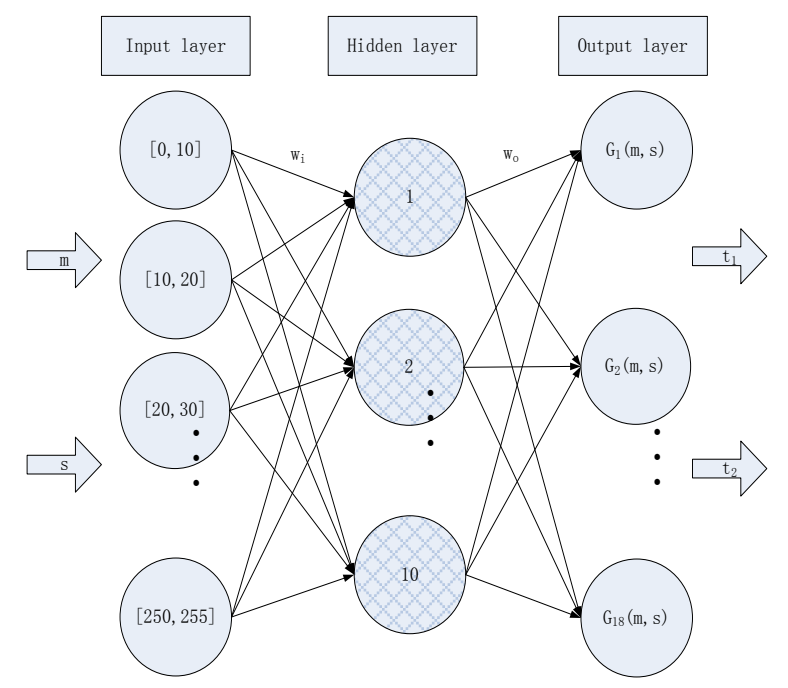

Fig. 3. The logic indicate of neural network

After created the initial neural network, extract the mean of image gray, the maximum gray deviation of image and corresponding mapping relation from the pre-sampled image as training and testing set of sample space (gross is 343 items). Randomly select 300 records as training set from sample space, the rest of 43 records as testing set. In this paper, the learning rate of neural network is 0.5 , the momentum factor is 0.01 . Using the training set to train the initial neural network, then using testing set to test it; the final neural network show good selective result of threshold function, and use dual-threshold function $\mathrm{G}(\mathrm{m}, \mathrm{s})$ to calculate the upper and lower threshold; has good image segmentation performance for testing image.

\section{EXPERIMENTAL DATA ANALYSIS}

Fig. 4 is the original image captured by Manta G-201B type of CCD industry camera in actual project; it required to measure the rotation angle of the hexagonal nut which is underneath the wheel based on machine vision. After analysis then convert this solving rotation angle problem to recognition a valid lateral surface of hexagonal nut in any rotation angle situation, which is using image processing method to dynamically segment effective lateral facet from image. Because in different rotation angle of the measured object situation on this problem, the formed by reflecting environment light image of measured object is dynamically uncertainly, so this paper proposed dual-threshold image segmentation method based on DTBNN algorithm, and through MATLAB simulation this method compared with dual-threshold of OTSU method and dual-threshold of maximum entropy method. 


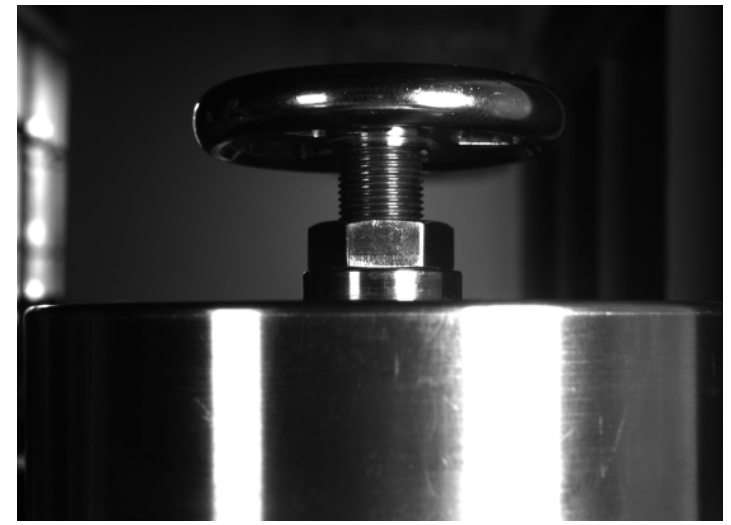

Fig. 4. Original image captured by CCD camera

Firstly, using division segmentation area separate measured hexagonal nut image from original integral image, the figure of separated image shown as Fig. 5(a) and Fig. 5(e), the figure shown acquisition images of measured hexagonal nut after rotate random angle. After graying of image which is separated out, could obtain its histogram. Then apply the method of this paper proposed, dual-threshold method of OTSU and dual-threshold method of maximum entropy to segment the original image by dual-threshold, its segmentation results shown in Fig.5, which Fig. 5(a) and Fig. 5(e) of gray histogram shown in Fig.6.
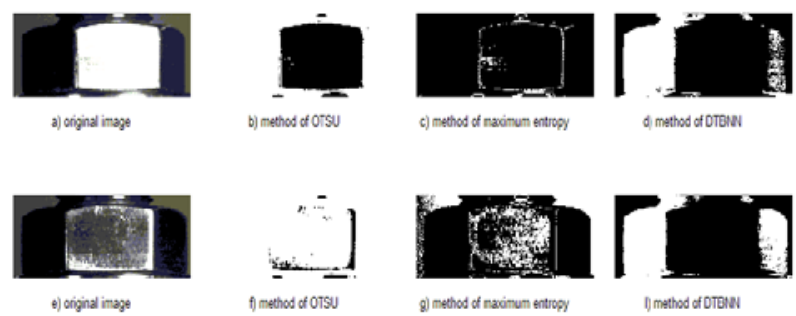

Fig. 5. The image processing contrast of experimental results: a and e are the measured image captured in two random rotation, $\mathrm{b}$ and $\mathrm{f}$ are the segmentation image using dual-threshold image segmentation method of OTSU, c and g are the segmentation image using dual-threshold image segmentation method of maximum entropy, $\mathrm{d}$ and $\mathrm{l}$ are the segmentation image using dual-threshold image segmentation method of DTBNN proposed by this paper
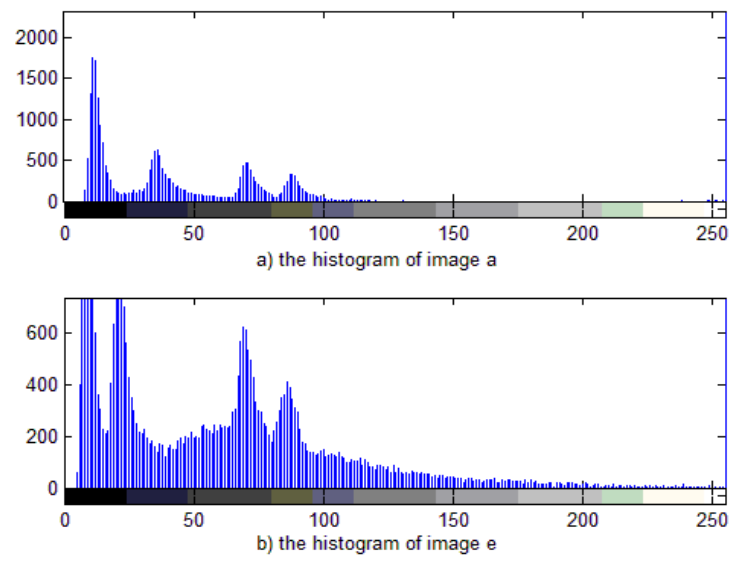

Fig. 6. The histogram of experimental image: a) is the histogram of original image Fig. 5 (a), b) is the histogram of original image in Fig. 5 (b)
Using dual-threshold image segmentation method of OTSU on Fig. 5(a) to obtain the upper and lower threshold of grayscale are 203 and 206, separate the bright and clear facet of hexagonal nut from the original image as Fig. 5(b); using dual-threshold image segmentation method of maximum entropy on Fig. 5(a) to obtain the upper and lower threshold of grayscale are 110 and 241, the edge of bright facet of hexagonal nut is taken out as Fig. 5(c); using dual-threshold image segmentation method of DTBNN proposed in this paper on Fig. 5(a) to obtain the upper and lower threshold of grayscale are 1 and 35, this method could explicitly segment out the lateral facet of hexagonal nut as Fig. 5(d). In the case of Fig. 5(a), according to the histogram of corresponding to Fig. 6(a) see that, there are obvious characters in the histogram of image, the image could be segmented effectively by three dual-threshold methods mentioned above all, so using three methods could extract effective lateral edge or surface and calculate out the rotation angle of measured hexagonal nut Base on the angle of Fig. 5(a) randomly rotate hexagonal nut to a small angle, after captured and divided the measured image like Fig. 5(e) shown, in this case there is no explicit characters in the histogram. Adopting dual-threshold image segmentation method of OTSU on Fig. 5(e), could obtain the upper and lower threshold are 246 and 249, and the threshold obtained by this method could not segment out the effective lateral edge or surface of hexagonal nut from measured image, shown as Fig. 5(f); using dual-threshold image segmentation method of maximum entropy to processing segmentation, could obtain the upper and lower threshold are 71 and 114, by obtained threshold could not segment out the effective lateral edge or surface as well, shown as Fig. 5(g); adopting the dualthreshold image segmentation method proposed by this paper to segment image on Fig. 5(e), obtain the upper and lower threshold are 1 and 25, it could segment out the lateral facet of hexagonal nut from measured image as shown in Fig. 5(l). Seen from the histogram of corresponding Fig. 6(b), there are no clear characters of histogram on Fig. 5(e), and there is little obvious changes comparing to Fig. 6(a), therefore the dualthreshold image segmentation method of OTSU and maximum entropy could not segment out effective object.

In addition, randomly extract three groups of test image records from 343 samples collected in experiments, each group contains 10, 20, 50 image samples respectively. Using dual-threshold image segmentation method of OTSU, dualthreshold image segmentation method of maximum entropy and dual-threshold image segmentation method of DTBNN proposed by this paper on each measurement image of testing groups, the statistics of segmentation results as shown in Tab. 1 and Tab. 2. The first column of table shows the number of test images in each sample group, the first row of table shows the using method of dual-threshold image segmentation, each record item respectively represent the accuracy and runtime on each methods. Using the number of correct segmentation image to divided into the total number of group samples calculate the accuracy; using the gross consuming time from start reading a sample image to complete segmentation all divided into the number of processed group samples to obtain the average running time. During dual-threshold image segmentation method of OTSU and dual-threshold image segmentation method of maximum entropy in dealing with 
experiments image data of project, which OTSU segmentation method could achieve 56\% correctly probability in processing, maximum entropy segmentation method could get $72 \%$ correctly probability in image processing. in these images which have no obvious feature of histogram, these two methods mentioned above could not correctly segment out the lateral edge or surface of hexagonal nut from measured image. For the proposed method in this paper, whether there is peek characteristic for the histogram of the image, could use the trained neural network to calculate out the segmentation threshold of image and segment out the lateral edge or surface of hexagonal nut correctly, the accuracy rate be close to $100 \%$. Meanwhile, analysis the statistics result from Tab. 2, the average runtime is $0.48 \mathrm{~s}$ by dual-threshold image segmentation method of OTSU, the average runtime is $1.59 \mathrm{~s}$ by dual-threshold image segmentation method of maximum entropy. Compared with dual-threshold image segmentation method of DTBNN adopted by this paper, due to before train the neural network then to use the DTBNN method, so this method which adopt training better neural network segmentation the image take 0.13 s for average runtimes, is better and faster than dual-threshold image segmentation of OTSU and maximum entropy. It is shown that the dualthreshold image segmentation method of this paper proposed has more operational efficiency than dual-threshold image segmentation method of OTSU and maximum entropy, and could be fit for dynamic image processing and extraction.

TABLE I. CORRECT NUMBER OF IMAGE SEGMENTATION

\begin{tabular}{cccc}
\hline $\begin{array}{c}\text { Number of image } \\
\text { sample }\end{array}$ & OTSU & $\begin{array}{c}\text { Maximum } \\
\text { entropy }\end{array}$ & DTBNN \\
\hline 10 & 5 & 8 & 10 \\
20 & 12 & 14 & 20 \\
50 & 29 & 33 & 50 \\
\hline
\end{tabular}

TABLE II. RUNTIME OF IMAGE SEGMENTATION(S)

\begin{tabular}{crcc}
\hline $\begin{array}{c}\text { Number of image } \\
\text { sample }\end{array}$ & OTSU & $\begin{array}{c}\text { Maximum } \\
\text { entropy }\end{array}$ & DTBNN \\
\hline 10 & 4.95 & 16.52 & 1.39 \\
20 & 9.75 & 31.64 & 2.64 \\
50 & 22.89 & 76.22 & 6.48 \\
\hline
\end{tabular}

\section{CONCLUSION}

Applied the neural network classification method based on the decision tree on threshold segmentation field of image processing, through the experiments verify that it could be used to dynamic image processing and obtain suitable dualthreshold by its classification ability of decision. In the actual project, it could satisfy with the requirement of practical problem and well resolve the project problem as well. The method of dual-threshold dynamic image segmentation based on DTBNN algorithm for segmentation rotation hexagonal nut in the practical project, could quickly and accurately segment out the effective lateral edge or surface of rotational hexagonal nut, has more advantages than dual-threshold image segmentation method of OTSU and maximum entropy which required peek feature in histogram. Verified by experiments, there is no effect on the method mentioned in this paper for the dynamic image which have obvious feature or not in histogram. Because of the method for constructing the neural network based on classification decision tree make it possible to use fewer training sample obtained a stable neural network.

\section{References}

[1] Ding Rui, Liu Jiajia, Li Bailin. Modified multilevel thresholding Otsu method for image segmentation [J]. Journal of Computer Applications, 2013, 33(S1): 214-217

[2] Tong xiaonian, Shi bo, Wang jiangqing. Dual-threshold Image Segmentation Method Based on QPSO Algorithm [J]. Journal of Sichuan University (Engineering Science Edition), 2010, 42(03): 132 138

[3] Zhang Chunli. Double Threshold Image Segmentation Based on Twolayer Genetic Algorithm [J]. Microcomputer Applications, 2008, 29(01):92-95

[4] Tao Wenbing, Liu Liman, Tian Jinwen. Image Segmentation by Double Thresholding with Maximum Fuzzy Entropy and Genetic Algorithm [J]. Signal Processing, 2005, 21(06): 684-687

[5] Zhou Xiancheng, Shen Quntai, Wang Junnian. Double Thresholding Algorithm for Image Segmentation based on Maximum Fuzzy Entropy and Particle Swarm Optimization [J]. Computer Engineering and Design, 2008, 29(12): 3188-3190

[6] Liu Xinxin, Li Xue, Wang Qiong. A Multi-Threshold Segmentation Method based on Gray-scale Histogram [J]. Computer Applications and Software, 2013, 30(12): 28-30

[7] Li Kangshun,, Li Kai, Zhang Wensheng. PCA Face Recognition Algorithm based on Improved BP Neural Network [J]. Computer Applications and Software, 2014, 31(01): 158-161

[8] Zhang Kai, Li Xuzhi. An Image Fusion Method based on Regional Correlation for High Resolution Remote Sensing Images [J]. Journal of Computer-Aided Design \& Computer Graphics, 2014, 26(06): 889-895

[9] I. K. Sethi. Neural Implementation of Tree Classifier [J]. IEEE Trans. Systems, Man, and Cybernetics, 1995, 25(8):1243-1249

[10] Li Aijun, Luo Siwei, Huang Hua. Decision Tree Based Neural Network Design [J]. Journal of Computer Research and Development, 2005, 42(08): 1312-1317

[11] Liu Dong, Feng Yong, Zhao Xianghui. Determination of Minimal Artificial Neural Network based on Decision Tree Algorithm[J]. Journal of Computer Applications, 2010, 30(Suppl.1): 83-85 